\title{
Hypoxia suppresses Kv 2.1 channel expression through endogenous 15-hydroxyeicosatetraenoic acid in rat pulmonary artery
}

\author{
Lei Guo - Zhaoping Qiu • Lei Zhang • \\ Shuo Chen • Daling Zhu
}

Received: 23 March 2010/ Accepted: 16 July 2010/Published online: 3 August 2010

(C) The Physiological Society of Japan and Springer 2010

\begin{abstract}
We have previously reported that hypoxia activates lung 15-lipoxygenase (15-LOX), which catalyzes arachidonic acid to produce 15-HETE, leading to constriction of neonatal rabbit pulmonary arteries. Hypoxia suppresses Kv2.1 channel expression. Although the Kv channel inhibition by hypoxia is likely to be mediated through 15-HETE, direct evidence is still lacking. To explore whether 15-LOX/15-HETE pathway contributes to the hypoxia-induced down-regulation of Kv2.1 channel, we performed studies using 15-LOX blockers, semi-quantitative PCR and western blot analysis. We found that Kv2.1 channel expression at the mRNA and protein levels was greatly up-regulated in pulmonary arterial smooth muscle cells (PASMCs) and pulmonary artery (PA) after blockade of endogenous 15-HETE under hypoxic condition. 15-HETE further decreased Kv2.1 channel expression in comparison with 12-HETE and 5-HETE in cultured PASMCs and PA under normoxic conditions. These data indicate that hypoxia suppresses Kv2.1 channel expression through endogenous 15-HETE in PA.
\end{abstract}

Keywords Kv2.1 $\cdot$ 15-HETE $\cdot$ 5-HETE - 12-HETE . Hypoxia

L. Guo $\cdot$ Z. Qiu $\cdot$ L. Zhang $\cdot$ S. Chen $\cdot$ D. Zhu $(\bowtie)$

Department of Biopharmaceutical Sciences,

College of Pharmacy, Harbin Medical University,

157 Baojian Road, Nangang District,

Harbin 150081, Heilongjiang, China

e-mail: dalingz@yahoo.com

D. Zhu

Biopharmaceutical Key Laboratory of Heilongjiang Province, Harbin 150081, China

\section{Introduction}

Hypoxic pulmonary vasoconstriction is a critical physiological mechanism that directs blood flow away from poorly ventilated regions in the lung to maintain an optimal ventilation-perfusion ratio for maximal oxygenation of the venous blood in the pulmonary artery (PA) [1-3]. One of the potential mechanisms involved in hypoxic pulmonary vasoconstriction is hypoxia-mediated inhibition of voltagegated $\mathrm{K}^{+}(\mathrm{Kv})$ channels in pulmonary arterial smooth muscle cells (PASMCs) [4-6]. In vascular smooth muscle, $\mathrm{K}^{+}$channels play an important role in the regulation of the resting membrane potential [7, 8]. Hypoxia inhibits the PASMC outward $\mathrm{K}^{+}$current [4-6, 9, 10], leading to membrane depolarization [4-6, 9] and constriction of small PA [9]. Therefore, much attention has been focused on identifying the $\mathrm{K}^{+}$channels involved in this response. However, how the $\mathrm{K}^{+}$channels are inhibited after hypoxia remains elusive. Both direct and indirect effects have been proposed for the channel inhibition. In the carotid body and pulmonary neuroepithelium, similar $\mathrm{K}^{+}$channels are inhibited by hypoxia directly, through molecular $\mathrm{O}_{2}$ [11]. There is evidence suggesting that LOX and their products play a role [12] in the process.

We have previously found that hypoxia up-regulates the activity of 15-LOX in PASMCs. The conversion of arachidonic acid to 15-HETE, a product of 15-LOX, is enhanced in microsomal and cytosolic fractions of PA from hypoxic neonatal rabbits. Exogenous 15-HETE causes robust and concentration-dependent contractions of PA rings under hypoxic conditions, which could be blocked by inhibition of endogenous 15-HETE production with lipoxygenase inhibitor [12]. Furthermore, blockade of $\mathrm{Kv}$ channels by 4-AP inhibits the 15-HETE-induced constriction of pulmonary arterial rings in hypoxic rabbits and rats 
[12-15]. 15-HETE decreases $\mathrm{Kv}$ currents $\left(I_{\mathrm{K}}\right)$ in freshly dispersed rabbit and rat PASMCs and raises $\left[\mathrm{Ca}^{2+}\right]_{\mathrm{i}}$ level in PASMCs [15]. We also have evidence that 15-HETE down-regulates Kv2.1 channel expression and inhibits $I_{\mathrm{K}}$ [16]. Although these studies indirectly suggest that hypoxia suppresses $\mathrm{Kv}$ channels via a 15-HETE-mediated mechanism, direct evidence remains unavailable.

In the present study, Kv2.1 channel expression at mRNA and protein levels was studied in PASMCs and PA after inhibition of 15-LOX. Our results suggest that hypoxia down-regulates Kv2.1 channel expression through endogenous 15-HETE. 15-HETE was found to be more potent than 5-HETE and 12-HETE in down-regulation Kv2.1 channel expression. These results demonstrate the link between 15-LOX, 15-HETE formation, and pulmonary vasoconstriction under hypoxic conditions.

\section{Materials and methods}

Reagents and instruments

15-HETE, 5-HETE, 12-HETE, cinnamyl 3, 4- dihydroxy[alpha]-cyanocinnamate (CDC), nordihydro-guiairetic acid (NDGA) were all purchased from Cayman Chemical (Michigan, USA). Anti-potassium channel Kv2.1 antibody was purchased from Santa cruz (USA). RT-PCR kit was purchased from Invitrogen (California, USA). All other reagents were from common commercial sources.

\section{Animals}

Adult male Wistar rats (200-250 g) were from the Experimental Animal Center of Harbin Medical University, which is fully approved by the Institutional Animal Care and Use Committee (IACUC). The animals were housed in the Animal Research Center of Harbin Medical University, at a controlled ambient temperature of $22-24^{\circ} \mathrm{C}$ with $50 \%$ relative humidity and a 12 -h light-dark cycle.

\section{Culture of distal PA}

Male wistar rats (weight 200-250 g) were anesthetized with Saffan (7-12 $\mathrm{mg} \mathrm{kg}^{-1} \mathrm{~h}^{-1}$ ), and the heart and lungs were removed. Resistance (4th or 5th order intralobar) branches of PA were dissected, cleaned of connective tissue and cut open longitudinally. Rat distal PAs were dissected from the lungs with endothelial cells denuded, and the PAs were immediately cultured in DMEM with $20 \%$ serum for $60 \mathrm{~h}$ under the following conditions: (1) normoxic $\left(21 \% \mathrm{O}_{2} / 5 \% \mathrm{CO}_{2} /\right.$ balance $\left.\mathrm{N}_{2}\right)$, (2) hypoxic $\left(2.5 \% \mathrm{O}_{2} /\right.$ $5 \% \mathrm{CO}_{2} /$ balance $\mathrm{N}_{2}$ ), and (3) hypoxic with $50 \mu \mathrm{M}$ NDGA and $5 \mu \mathrm{M} \mathrm{CDC}$, for western blotting and RT-PCR.
Dissociation and culture of PASMCs

Primary cultures of PASMCs were prepared from rat pulmonary arteries [6]. The isolated distal arterial rings were incubated in Hanks' balanced salt solution containing $1.5 \mathrm{mg} / \mathrm{ml}$ of collagenase for $20 \mathrm{~min} \mathrm{[17].} \mathrm{After} \mathrm{incuba-}$ tion, a thin layer of the adventitia was carefully stripped off with fine forceps and the endothelium was removed by gently scratching the intimal surface with a surgical blade. The smooth muscles were then digested with $1.0 \mathrm{mg} / \mathrm{ml}$ of collagenase and $0.5 \mathrm{mg} / \mathrm{ml}$ of elastase (Sigma) for $60 \mathrm{~min}$ at $37^{\circ} \mathrm{C}$. The cells were plated onto $25 \times 25 \mathrm{~mm}$ coverslips in $10-\mathrm{cm}$ petri dishes (for molecular biological experiments) in $20 \%$ fetal bovine serum (FBS)-containing DMEM and cultured in a humidified incubator with $5 \%$ $\mathrm{CO}_{2}$ for $3-5$ days at $37^{\circ} \mathrm{C}$. Before each experiment, the cells were incubated in $0.3 \%$ FBS-DMEM for $12-24 \mathrm{~h}$ to stop cell growth.

The purity of PASMCs in the primary cultures was confirmed by specific monoclonal antibody raised against smooth muscle $\alpha$-actin. The total number of primary cultured cells was estimated with membrane-permeable $4^{\prime}, 6^{\prime}$ diamidino-2-phenylindole (DAPI, $5 \mu \mathrm{M}$; Molecular Probes) for nucleic acid stain. All the DAPI-stained cells were cross-reacted with SMC $\alpha$-actin antibodies, indicating that the cells were pure PASMCs.

\section{Western blot procedures}

Cultured rat distal PA and PASMC were gently washed twice in cold PBS, put into $0.3 \mathrm{ml}$ of lysis buffer $(1 \%$ Nonidet P-40, $0.5 \%$ sodium deoxycholate, $0.1 \%$ SDS, $100 \mu \mathrm{g} / \mathrm{ml}$ of phenylmethylsulfonyl fluoride, and $30 \mu \mathrm{M}$ of aprotinin), and incubated for $30 \mathrm{~min}$ on ice. The lysates were then sonicated and centrifuged at $16,099 \mathrm{~g}$ for $10 \mathrm{~min}$, and the insoluble fraction was discarded. The protein concentrations in the supernatant were determined with the bicinchoninic acid protein assay (Pierce, Rockford, IL, USA) with bovine serum albumin (BSA) as standard. Fifty micrograms of protein were mixed and boiled in SDSPAGE sample buffer for $5 \mathrm{~min}$. The proteins fractionated by $7.5 \%$ SDS-PAGE were then transferred to nitrocellulose membranes by electroblotting in a Mini Trans-Blot cell transfer apparatus (Bio-Rad) under conditions recommended by the manufacturer. After incubation overnight at $4^{\circ} \mathrm{C}$ in a blocking buffer $(0.1 \%$ Tween 20 in PBS $)$ containing 5\% nonfat dry milk powder, the membranes were incubated with affinity-purified rabbit polyclonal antibodies specific for Kv2.1 (Sigma). The monoclonal antibody specific for smooth muscle $\beta$-actin (Sigma) was used as an internal control. The membranes were then washed and incubated with anti-rabbit horseradish peroxidaseconjugated IgG for $120 \mathrm{~min}$ at room temperature. The 
bound antibodies were detected with an enhanced chemiluminescence detection system (Amersham, USA).

\section{RT-PCR}

Sequences of rat Kv2.1 cDNA were obtained from the GenBank $^{\mathrm{TM}}$ data base (Accession \# X16476). Accordingly, sense primer, 5'-GGGTTGCTAGTGAAAGAGTG G-3', and anti-sense primer, 5'-CATACGAGCGTTGTTTGGA$3^{\prime}$, were designed for a 321-bp fragment. For $\beta$-actin control, sense primer, 5'-CCGTAAAGACC TCTATGCC AACA-3', and anti-sense primer, 5'-CGGACTCATCGTACTCCTGCT- $3^{\prime}$, were used to obtain a 500-bp PCR fragment. Total RNA prepared from cultured rat distal PA and PASMC by the acid guanidinium thiocyanate-phenolchloroform extraction method was reverse transcribed with the Superscript First-strand cDNA Synthesis kit (Invitrogen). The fidelity and specificity of the sense and antisense oligonucleotides were examined with the BLAST program. The PCR products were amplified in a DNA thermal cycler, followed by electrophoresis through a $1 \%$ agarose gel. The amplified cDNA bands were then visualized with GelStar staining. To quantify the PCR products, an invariant mRNA of $\beta$-actin was used as an internal control. The OD values for the channel signals, measured by a Kodak electrophoresis documentation system, were normalized to the OD values for the $\beta$-actin signals; the ratios are expressed as arbitrary units for quantitative comparison [18].

\section{Statistical analysis}

All values are expressed as mean \pm SEM. The significance of mean values among the experimental groups was calculated by using two-tailed analyses of variance (ANOVA) followed by Dunnett's test. Differences were considered to be significant if $P \leq 0.05$.

\section{Results}

Time-dependent changes in Kv2.1 channel expressions after hypoxia

The time-dependent changes in Kv2.1 channel expression protein and mRNA were studied by using western blot and RT-PCR analysis in rat PASMCs cultured under hypoxic conditions. We found that Kv2.1 channel protein expression decreased after $60 \mathrm{~h}$ of exposure to hypoxia. No significant change in expression was found within $48 \mathrm{~h}$ of exposure to hypoxia. However, the mRNA levels of Kv2.1 were decreased after $48 \mathrm{~h}$ of exposure to moderate hypoxia, and the reduction progressed further at $60 \mathrm{~h}$ (Fig. 1).
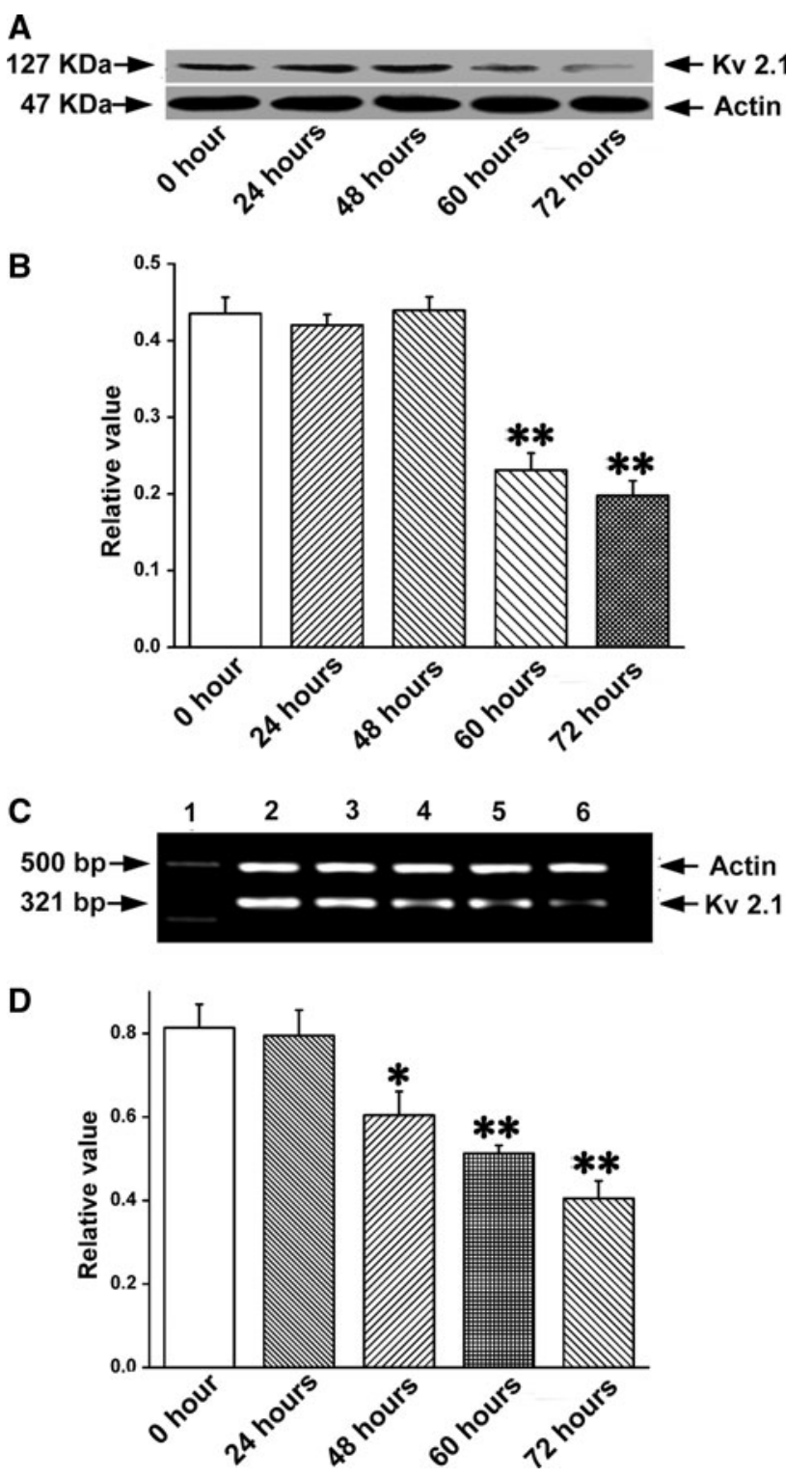

Fig. 1 Time-course of Kv2.1 channel expression in cultured rat PASMCs exposured to hypoxia. a Western blotting of Kv2.1 channel expression in PASMCs at different time point. b Densitometric analysis of the western blot $(n=4, * * P<0.01)$. c PCR-amplified products were displayed in agarose gels stained with ethidium bromide for Kv2.1 (321 bp) and $\beta$-actin (500 bp) transcripts. Lane 1 Marker, Lane 20 h, Lane 324 h, Lane 448 h, Lane 560 h, Lane 6 $72 \mathrm{~h}$. d Densitometric analysis of the RT-PCR $(n=4, * * P<0.01$, $* P<0.05)$

The effects of hypoxia and endogenous 15-HETE on Kv2.1 channel expression in cultured rat distal PA

If the hypoxia-induced down-regulation of Kv2.1 channel expression is mediated through 15-HETE, inhibition of endogenous 15-LOX/15-HETE should recover Kv2.1 channel expression. To test this hypothesis, western blot analysis and RT-PCR techniques were used to examine the contribution of endogenous 15-HETE to Kv2.1 channel 
expression in separated PA cultured under normoxic or hypoxic conditions for $60 \mathrm{~h}$ with or without lipoxygenases inhibitors, $\mathrm{CDC}(5 \mu \mathrm{M})$ or NDGA $(50 \mu \mathrm{M})$, respectively. The results show that inhibition of endogenous 15-LOX/15-HETE significantly recovered Kv2.1 channel expression at both the protein and mRNA levels in cultured distal PA (Fig. 2).

Alleviation of the hypoxia-induced Kv2.1 channel down-regulation with 15-LOX blockade in cultured PASMCs and PA

To elucidate whether 15-LOX plays a role in the hypoxiainduced inhibition of Kv2.1 channel expression in cultured PASMCs and PA, we suppressed 15-LOX activity by using 15-LOX blockers. Inhibition of endogenous 15-HETE with $5 \mu \mathrm{M} \mathrm{CDC}$ relieved the inhibition of Kv2.1 channel downregulation caused by hypoxia. Similar results were obtained with another 15-LOX inhibitor NDGA $(50 \mu \mathrm{M})$.

If the alleviation of hypoxia-induced Kv2.1 channel down-regulation is mediated through 15-HETE, the effect should be diminished or blocked with addition of exogenous 15-HETE. To test this possibility, $1 \mu \mathrm{M}$ 15-HETE was added to the cultured PASMC and PA, and Kv2.1 channel expression was examined with or without 15-LOX blockers. The alleviation of hypoxia-induced Kv2.1 channel inhibition by $5 \mu \mathrm{M}$ CDC or $50 \mu \mathrm{M}$ NDGA, respectively, was significantly attenuated in the presence of exogenous 15-HETE. Without inhibition of endogenous 15-HETE formation, hypoxia produced an even greater inhibitions of Kv2.1 channel expression in the presence of exogenous 15-HETE, suggesting that both endogenous and exogenous 15-HETEs have inhibitory effects on Kv2.1 channel expression (Figs. 3, 4).

\section{Effects of other HETEs isoforms}

The specificity of 15-HETE in causing the down-regulation of Kv2.1 channel expression in cultured rat PASMCs and PA was examined by using both exogenous 5-HETE and 12-HETE. Under normoxic conditions, 12-HETE inhibited Kv2.1 channel expression in cultured PASMCs and PA. 12-HETE, 5-HETE was found to be less potent than 15-HETE in causing inhibition of the Kv2.1 channel. These data suggest that 15-HETE is more potent than 12-HETE, 5-HETE in down-regulation of Kv2.1 expression (Fig. 5).

\section{Discussion}

We have demonstrated that 15 -LOX pathway via its metabolites, 15-HETE, plays a role in the hypoxia-induced pulmonary arterial vessel constriction. We have further proved that Kv1.5, Kv2.1, and Kv3.4 channels contribute
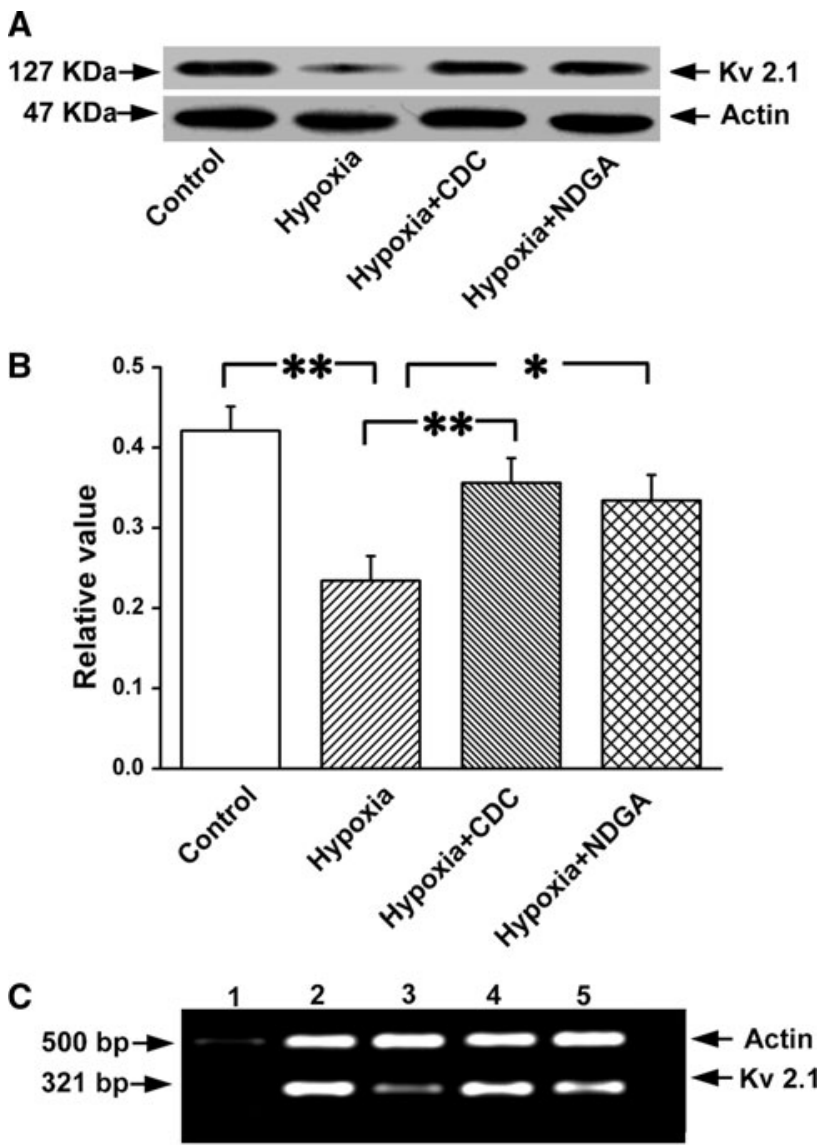

D

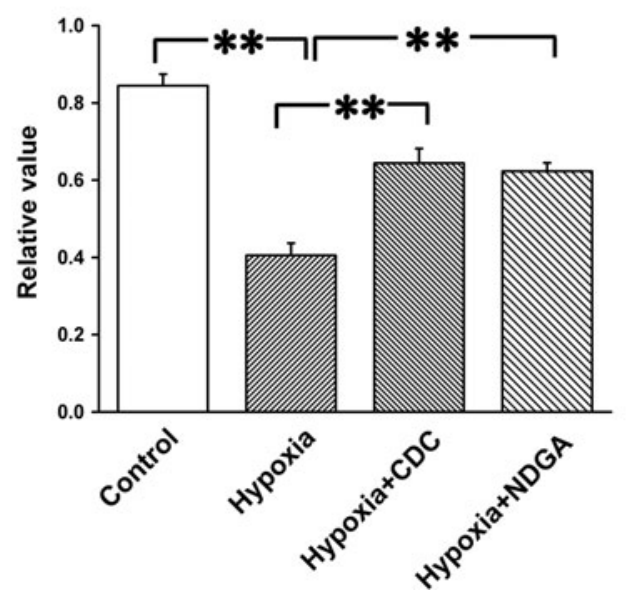

Fig. 2 Hypoxia-induced down-regulation of Kv2.1 channel in cultured rat pulmonary artery was partly restored by 15 -LOX inhibitors CDC $(5 \mu \mathrm{M})$ and NGDA $(50 \mu \mathrm{M})$. a Western blotting of Kv2.1 channel. b Densitometric analysis of the western blot $(n=4, * * P<0.01$, $* P<0.05)$. c PCR-amplified products were displayed in agarose gels stained with ethidium bromide for Kv2.1 channel (321 bp) and $\beta$-actin (500 bp) transcripts. Lane 1 Marker, Lane 2 control, Lane 3 hypoxia, Lane 4 hypoxia + CDC, Lane 5 hypoxia + NDGA. d Densitometric analysis of the RT-PCR. $\left(n=4\right.$, $\left.{ }^{*} * P<0.01,{ }^{*} P<0.05\right)$

to the 15-HETE-induced pulmonary vasoconstrictions $[15,16]$. In the present study, we have examined the effects of endogenous 15-HETE on Kv2.1 channel expression in 

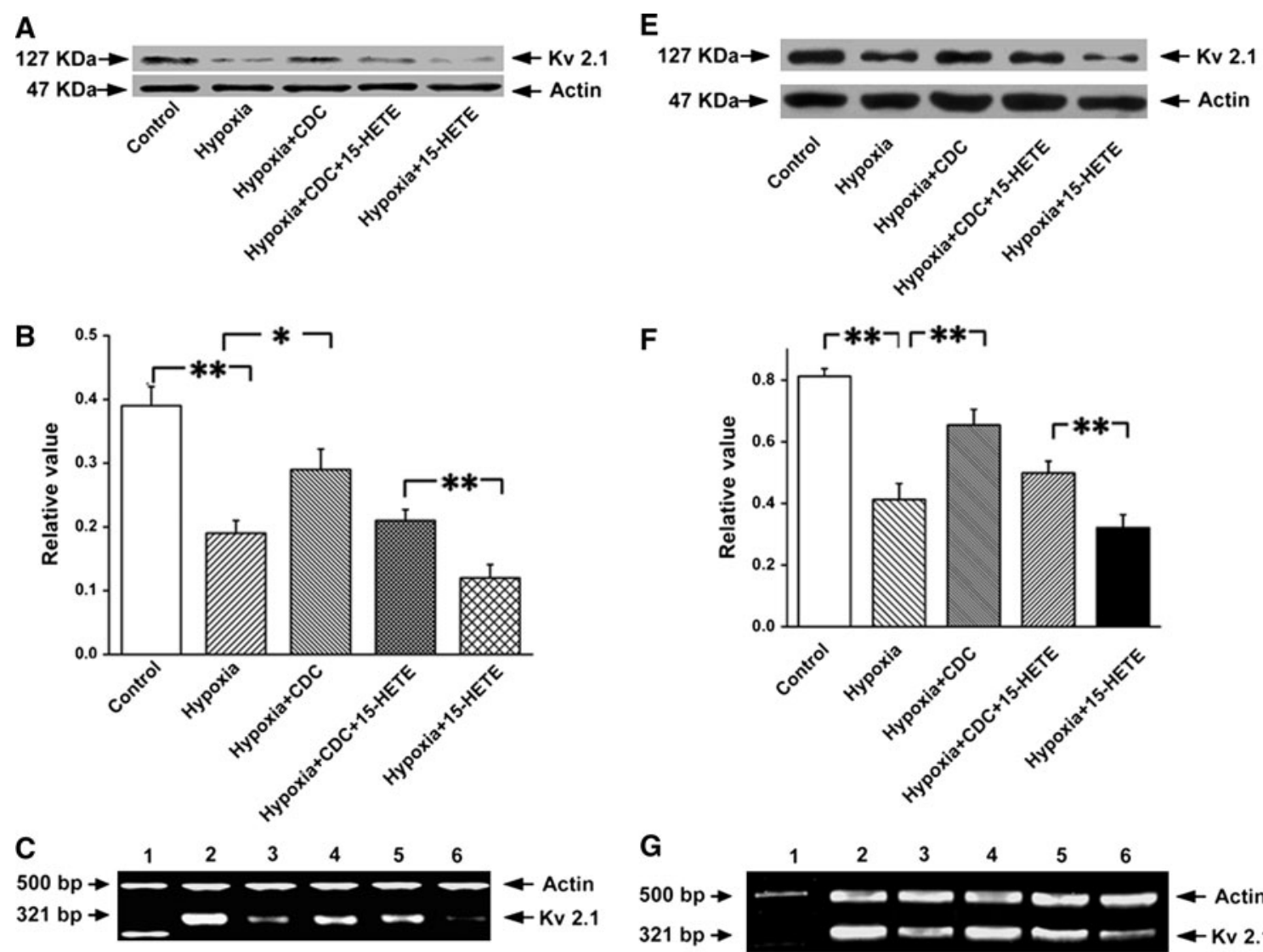

$\mathbf{F}$
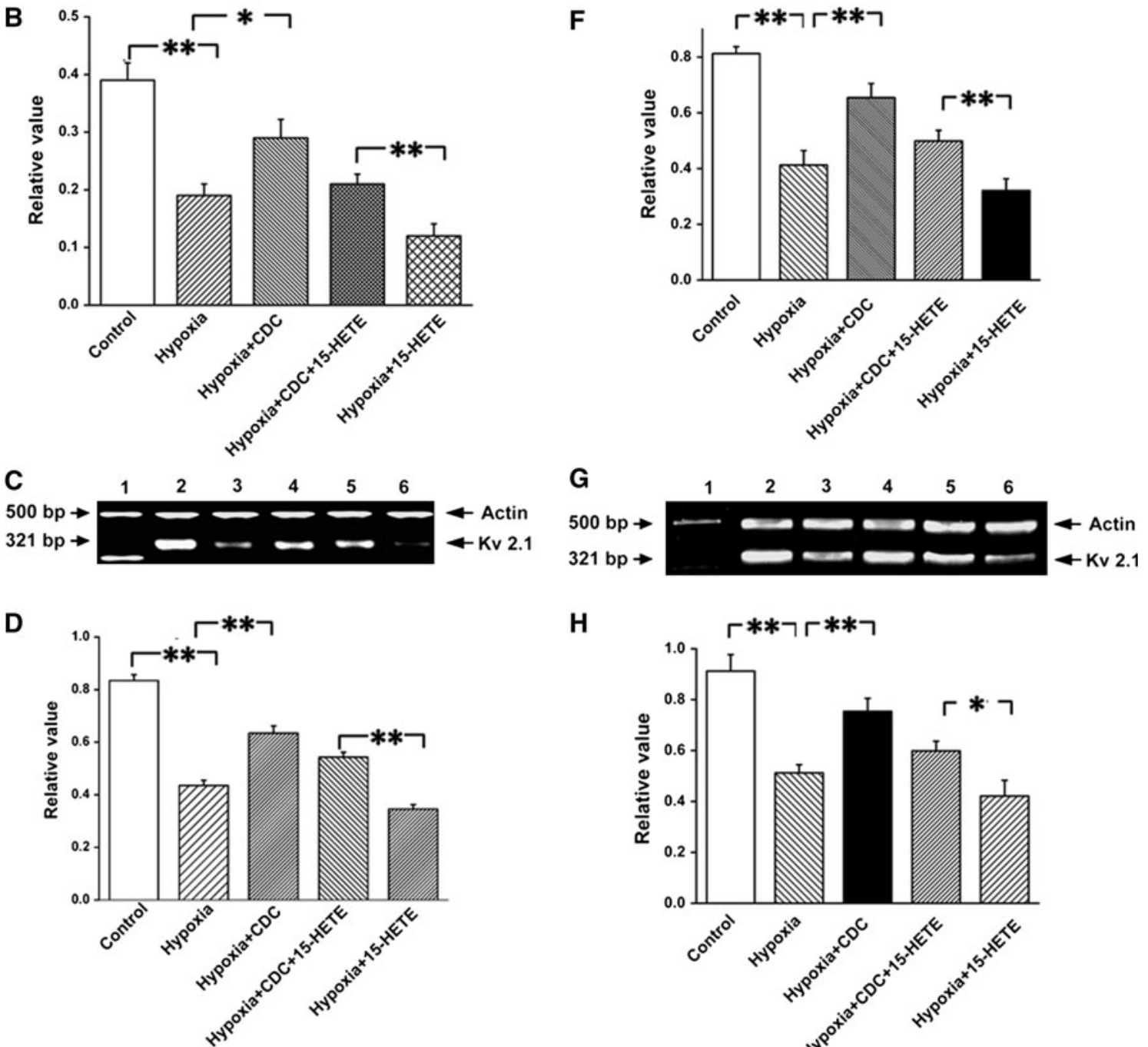

H

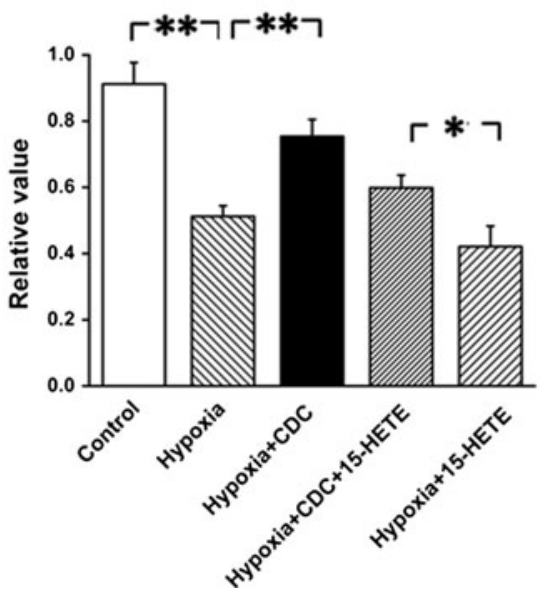

Fig. 3 Hypoxia-induced down-regulation of Kv2.1 channel expression in cultured rat PASMCs and PA were partly restored by 15 -LOX inhibitor CDC $(5 \mu \mathrm{M})$ under hypoxia. a Western blotting of Kv2.1 channel expression in PASMCs. b Densitometric analysis of the western blot $(n=4, * * P<0.01, * P<0.05)$. c PCR-amplified products were displayed in agarose gels stained with ethidium bromide for Kv2.1 (321 bp) and $\beta$-actin (500 bp) transcripts. Lane 1 Marker, Lane 2 control, Lane 3 hypoxia, Lane 4 hypoxia $+\mathrm{CDC}$, Lane 5 hypoxia + CDC +15 -HETE, Lane 6 hypoxia +15 -HETE.

freshly isolated PASMCs and PA. The results indicate that hypoxia, via endogenous 15-HETE, inhibits Kv2.1 channel expression.

d Densitometric analysis of the RT-PCR $(n=4, * * P<0.01$, $* P<0.05$ ). e Western blotting of Kv2.1 channel expression in cultured rat PA. $\mathbf{f}$ Densitometric analysis of the western blot $(n=4$, $\left.* * P<0.01,{ }^{*} P<0.05\right)$. g PCR-amplified products were displayed in agarose gels stained with ethidium bromide for Kv2.1 (321 bp) and $\beta$-actin (500 bp) transcripts. Lane 1 Marker, Lane 2 control, Lane 3 hypoxia, Lane 4 hypoxia $+\mathrm{CDC}$, Lane 5 hypoxia $+\mathrm{CDC}+15$ HETE, Lane 6 hypoxia +15 -HETE. h Densitometric analysis of the RT-PCR $(n=4, * * P<0.01, * P<0.05)$

At least four different major families of $\mathrm{K}^{+}$channels have been identified in single PASMCs: (1) Kv channels, (2) large conductance $\mathrm{Ca}^{2+}$-activated $\mathrm{K}^{+}$channels 

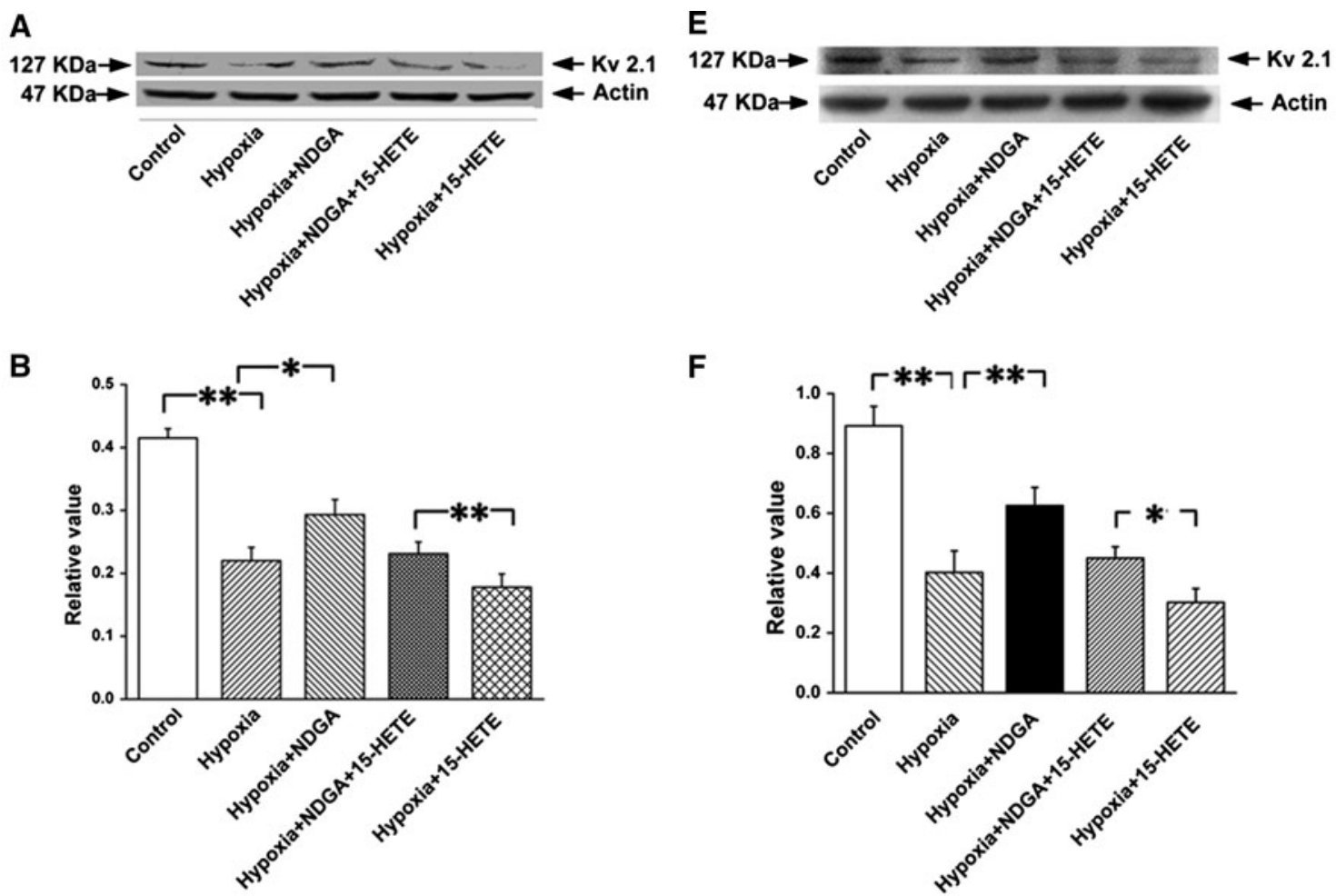

$\mathbf{F}$
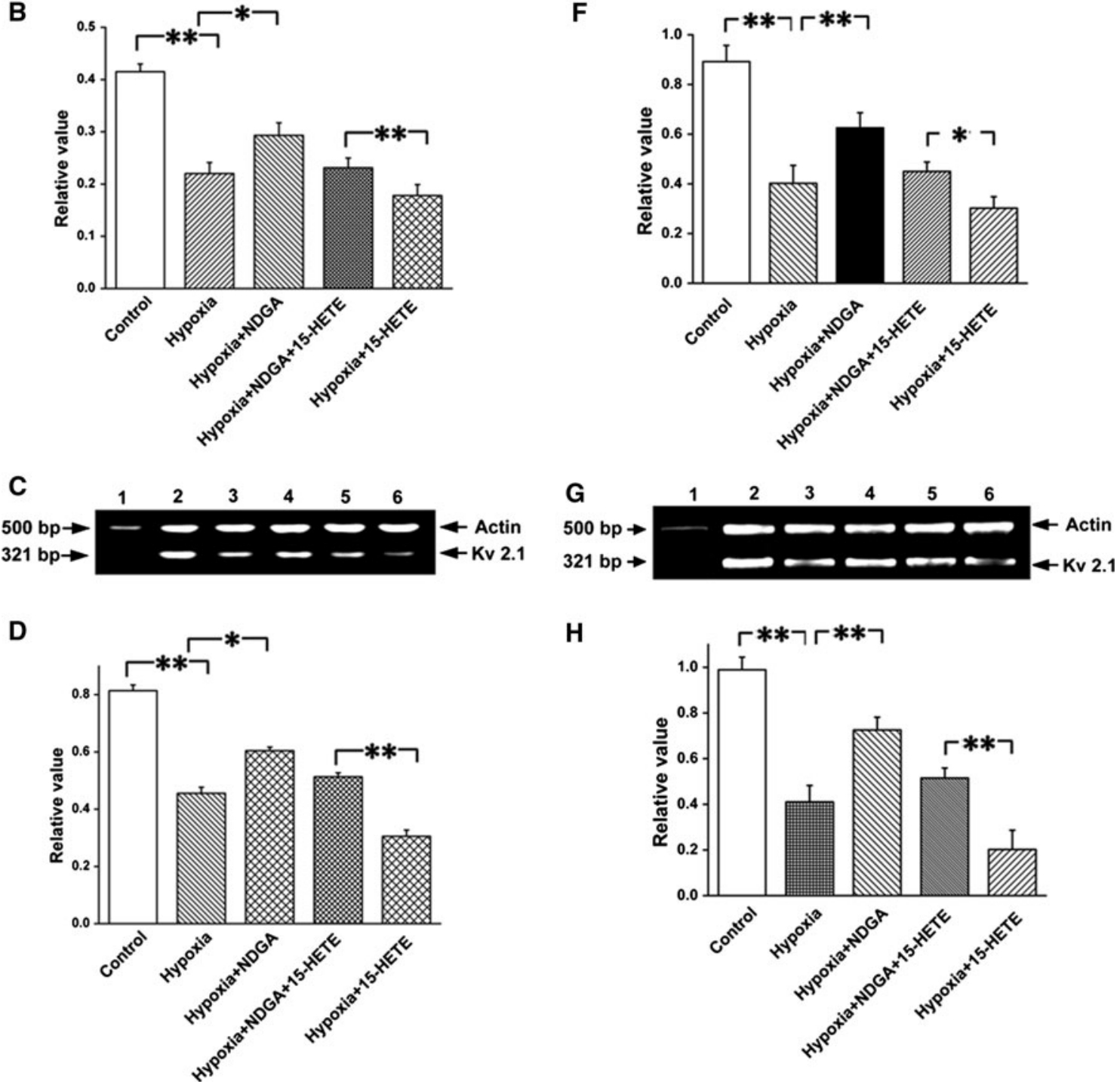

H

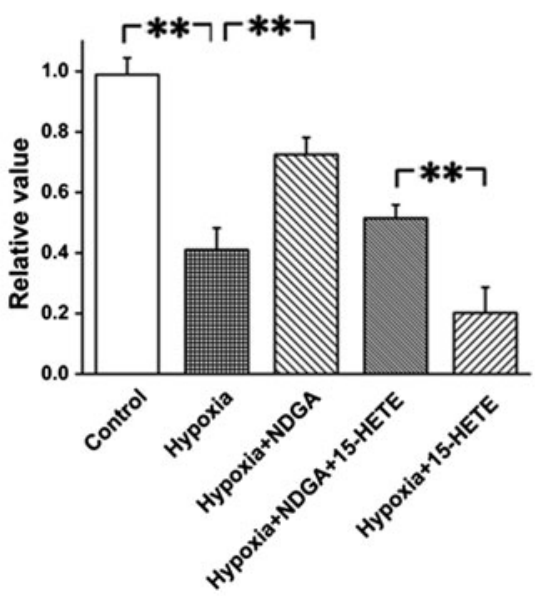

Fig. 4 Hypoxia-induced down-regulation of Kv2.1 channel expression in cultured rat PASMCs and PA were partly restored by 15-LOX inhibitor NGDA $(50 \mu \mathrm{M})$ under hypoxic conditions. a Western blotting of Kv2.1 channel expression in PASMCs. b Densitometric analysis of the western blot $\left(n=4,{ }^{*} P<<0.01,{ }^{*} P<0.05\right)$. c PCRamplified products were displayed in agarose gels stained with ethidium bromide for $\mathrm{Kv} 2.1$ (321 bp) and $\beta$-actin (500 bp) transcripts. Lane 1 Marker, Lane 2 control, Lane 3 hypoxia, Lane 4 hypoxia + NDGA, Lane 5 hypoxia + NDGA + 15-HETE, Lane 6

hypoxia +15 -HETE. d Densitometric analysis of the RT-PCR $(n=4, * * P<0.01, * P<0.05)$. e Western blotting of Kv2.1 channel expression in cultured rat PA. f Densitometric analysis of the western blot $\left(n=4\right.$, $\left.{ }^{*} P<0.01,{ }^{*} P<0.05\right)$. g PCR-amplified products were displayed in agarose gels stained with ethidium bromide for Kv2.1 (321 bp) and $\beta$-actin (500 bp) transcripts. Lane 1 Marker, Lane 2 control, Lane 3 hypoxia, Lane 4 hypoxia + NDGA, Lane 5 hypoxia + NDGA +15 -HETE, Lane 6 hypoxia +15 -HETE. $\mathbf{h}$ Densitometric analysis of the RT-PCR $(n=4, * * P<0.01, * P<0.05)$ 
A

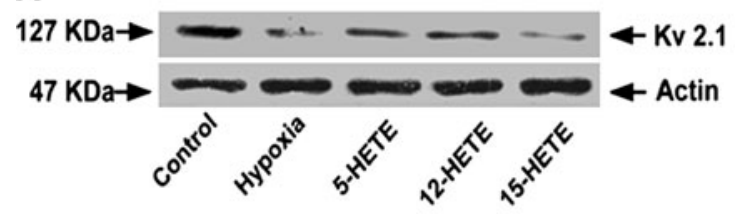

B

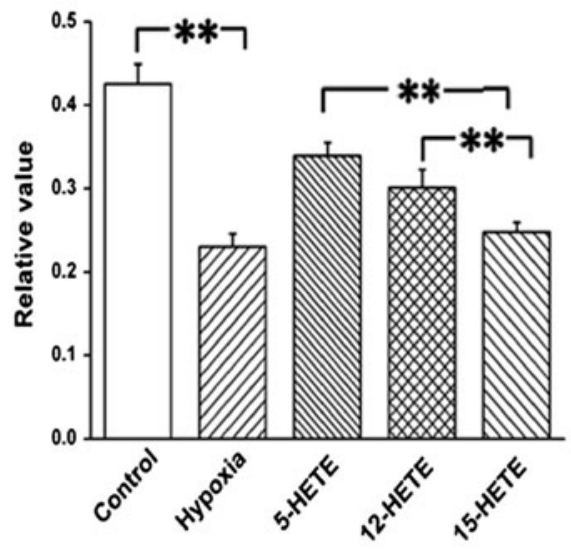

C

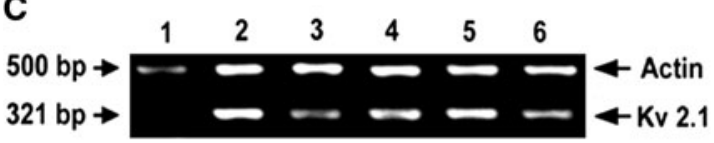

D

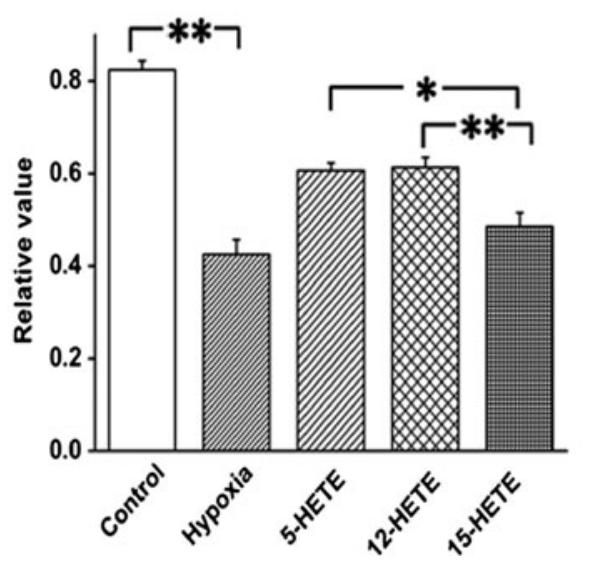

Fig. 5 Influence of exogenous 5-HETE $(1 \mu \mathrm{M})$, 12-HETE $(1 \mu \mathrm{M})$ and 15-HETE $(1 \mu \mathrm{M})$ on Kv2.1 channel expression in cultured rat PASMCs and PA under normoxic conditions. a Western blotting of Kv2.1 channel expression in PASMCs. b Densitometric analysis of the western blot $\left(n=4, * * P<0.01,{ }^{*} P<0.05\right)$. c PCR-amplified products were displayed in agarose gels stained with ethidium bromide for Kv2.1 (321 bp) and $\beta$-actin (500 bp) transcripts. Lane 1 Marker, Lane 2 control, Lane 3 hypoxia, Lane 4 5-HETE, Lane 512 HETE, Lane 6 15-HETE. d Densitometric analysis of the RT-PCR

(BKCa), (3) ATP-sensitive $\mathrm{K}^{+}$channels (KATP), and (4) inward rectifier $\mathrm{K}^{+}$channels (KIR). It is known that several members in the $\mathrm{Kv}$ channel family are sensitive to hypoxia, including Kv1.2 [19], Kv1.5 [20], Kv2.1 [21], Kv3.1b [22], Kv3.3 [23], and Kv4.2 [24]. Our previous studies have shown that 15-HETE suppresses Kv1.5, Kv2.1 and Kv3.4

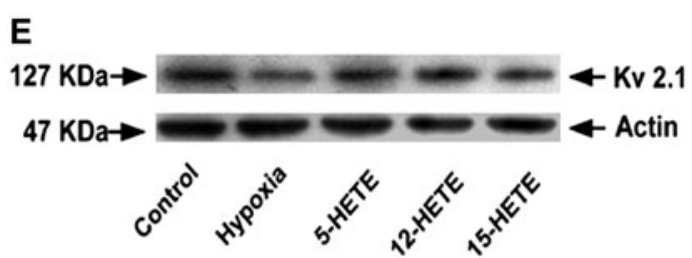

$\mathbf{F}$
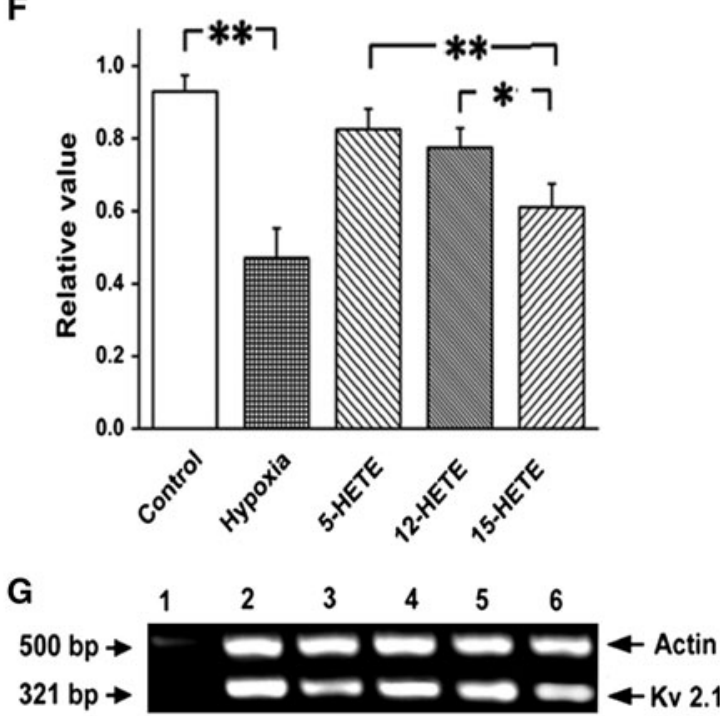

H

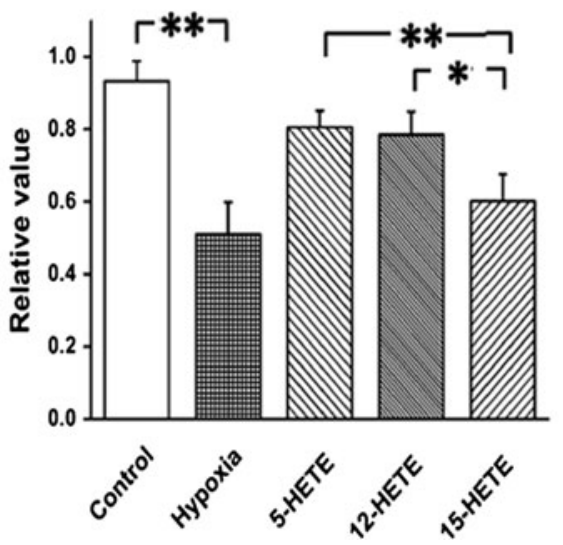

$(n=4, * * P<0.01, * P<0.05)$. e Western blotting of Kv2.1 channel expression in cultured rat PA. $\mathbf{f}$ Densitometric analysis of the western blot $(n=4, * * P<0.01, * P<0.05)$. g PCR-amplified products were displayed in agarose gels stained with ethidium bromide for Kv2.1 (321 bp) and $\beta$-actin (500 bp) transcripts. Lane 1 Marker, Lane 2 control, Lane 3 hypoxia, Lane 4 5-HETE, Lane 5 12-HETE, Lane 6 15-HETE. h Densitometric analysis of the RT-PCR $(n=4$, $* * P<0.01, * P<0.05)$

channels expressions, and inhibits IK in PASMCs. Similar results were observed in PASMCs maintained in subacute hypoxic environment [16].

Although exogenous 15-HETE, with a similar effect to hypoxia, inhibited Kv1.5, Kv2.1 and Kv3.4 channels expressions and the whole-cell $\mathrm{K}^{+}$currents [16], no direct 
evidence was available to fill the gap in our knowledge of how hypoxia induces membrane depolarization and $\mathrm{Kv}$ channel inhibition. We assumed that hypoxia inhibits Kv2.1 channel expression through 15-HETE-mediated mechanism. If this is the case, blockade of endogenous 15-HETE formation by lipoxygenase inhibitors might be able to rescue Kv2.1 channel expression. Likewise, exogenous 15-HETE would decrease Kv2.1 channel expression in cultured PASMCs pretreated with lipoxygenase inhibitors under hypoxic conditions, but Kv2.1 channel expression should be higher than that of PASMCs treated only with 15-HETE under hypoxic conditions without lipoxygenase inhibitors. Supporting the scenario, our results showed that Kv2.1 channel expression at both protein and mRNA levels in PASMCs was markedly increased after pretreatment with 15-LOX blockers. The expression of Kv2.1 channel in PASMCs pretreated with CDC or NDGA was greatly increased in response to exogenous 15-HETE compared with that of PASMCs without blocking of 15-LOX. These data provide further evidence that blockade of endogenous 15-HETE formation decreased the inhibiting effect of hypoxia on Kv2.1 channel expression.

Meanwhile, we wondered if what happened in PASMC might also happen in PA. In this study, we successfully established a hypoxic PA-culture procedure (PA model) to examine the $\mathrm{Kv}$ channel changes in cultured rat PA. The results showed that endogenous 15-HETE significantly inhibited Kv2.1 channel expression at both the protein and the mRNA levels, which were similar to the effect of hypoxia. The results from this PA study were similar to those from the animal model and the cultured PASMC [17]. Therefore, the PA study is an alternative way to repeat the experiment carried out in a hypoxic animal model and allows us to examine the effects of various agents on PA easily both morphologically and functionally.

The LOXs comprise a family of non-heme ironcontaining dioxygenases that catalyze, in a stereospecific manner, the oxygenation of the 5-, 12-, or 15-carbon atoms of arachidonic acid. There is a previous report that 5-LOX is involved in the regulation of lung vascular tone and in the development of chronic pulmonary hypertension in hypoxic rodent models [25]. 12-LOX and its product, 12(S)-HETE, are important intermediates in hypoxiainduced pulmonary arterial smooth muscle proliferation and may participate in hypoxia-induced pulmonary hypertension [26]. In the present study, we found that CDC and NDGA only partially restored the hypoxia-induced down-regulation of Kv2.1 channel. We speculated that other HETEs might also affect Kv2.1 channel expression. Our results showed that 5-HETE, 12-HETE and 15-HETE all have inhibiting effect on Kv2.1 channel expression in cultured PASMC under normoxic conditions, with 15-HETE the strongest. Thus it is likely that an array of LOXs is activated following hypoxia, with each affecting different target molecules, while the 15-LOX/15-HETE pathway may play a more important role in down-regulation of Kv2.1 channel expression induced by hypoxia.

In conclusion, we have demonstrated that blocking the formation of endogenous 15-HETE increased Kv2.1 expression in PASMCs and pulmonary artery under hypoxic conditions at both protein and mRNA levels. Inhibition of endogenous 15-HETE influences Kv2.1 expression affected by exogenous 15-HETE in PASMCs under hypoxic conditions. 15-HETE further decreased Kv2.1 channel expression compared with 12-HETE, 5-HETE in cultured PASMCs. All these data suggest that the decreased Kv2.1 channel expression, induced by hypoxia, is regulated by 15-HETE. These studies are the first effort to establish the molecular and ionic mechanisms of the hypoxia in inhibiting $\mathrm{Kv}$ channels and provide insight into the potential contribution of 15-HETE to the regulation of $\mathrm{K}^{+}$channel activity.

Acknowledgments This work was supported by the National Natural Science Foundation of China (No. 30370578 and No. 30470752); Harbin Medical University Innovation Foundation.

\section{References}

1. Fishman AP (1976) Hypoxia on the pulmonary circulation. How and where it acts. Circ Res 38:221-231

2. Staub NC (1985) Site of hypoxic pulmonary vasoconstriction. Chest 88:240S-245S

3. Voelkel NF (1986) Mechanisms of hypoxic pulmonary vasoconstriction. Am Rev Respir Dis 133:1186-1195

4. Post JM, Gelband CH, Hume JR (1995) $\left[\mathrm{Ca}^{2+}\right]_{\mathrm{i}}$ inhibition of $\mathrm{K}^{+}$ channels in canine pulmonary artery Novel mechanism for hypoxia-induced membrane depolarization. Circ Res 77:131-139

5. Post JM, Hume JR, Archer SL, Weir EK (1992) Direct role for potassium channel inhibition in hypoxic pulmonary vasoconstriction. Am J Physiol 262:C882-C890

6. Yuan XJ, Goldman WF, Tod ML, Rubin LJ, Blaustein MP (1993) Hypoxia reduces potassium currents in cultured rat pulmonary but not mesenteric arterial myocytes. Am J Physiol 264:L116L123

7. Jackson WF (2000) Ion channels and vascular tone. Hypertension 35:173-178

8. Nelson MT, Quayle JM (1995) Physiological roles and properties of potassium channels in arterial smooth muscle. Am J Physiol 268:C799-C822

9. Archer SL, Huang JM, Reeve HL et al (1996) Differential distribution of electrophysiologically distinct myocytes in conduit and resistance arteries determines their response to nitric oxide and hypoxia. Circ Res 78:431-442

10. Osipenko ON, Evans AM, Gurney AM (1997) Regulation of the resting potential of rabbit pulmonary artery myocytes by a low threshold, $\mathrm{O}_{2}$-sensing potassium current. $\mathrm{Br} \mathrm{J}$ Pharmacol 120:1461-1470

11. Ganfornina MD, Lopez-Barneo J (1992) Potassium channel types in arterial chemoreceptor cells and their selective modulation by oxygen. J Gen Physiol 100:401-426 
12. Zhu D, Medhora M, Campbell WB, Spitzbarth N, Baker JE, Jacobs ER (2003) Chronic hypoxia activates lung 15-lipoxygenase, which catalyzes production of 15-HETE and enhances constriction in neonatal rabbit pulmonary arteries. Circ Res 92:992-1000

13. Weissmann N, Seeger W, Conzen J, Kiss L, Grimminger F (1998) Effects of arachidonic acid metabolism on hypoxic vasoconstriction in rabbit lungs. Eur J Pharmacol 356:231-237

14. Pfister SL, Spitzbarth N, Nithipatikom K, Edgemond WS, Falck JR, Campbell WB (1998) Identification of the 11, 14, 15- and 11, 12, 15-trihydroxyeicosatrienoic acids as endothelium-derived relaxing factors of rabbit aorta. J Biol Chem 273:30879-30887

15. Han WN, Li XH, Jiang ZY et al (2004) Effect of 15-HETE on potassium channels of rabbit pulmonary arterial smooth muscles during hypoxia. Sheng Li Xue Bao 56:717-722

16. Li Q, Zhang R, Lu CL, Liu Y, Wang Z, Zhu DL (2006) The role of subtypes of voltage-gated $\mathrm{K}+$ channels in pulmonary vasoconstriction induced by 15 -hydroeicosatetraenoic acid. Yao Xue Xue Bao 41:412-417

17. Wang J, Weigand L, Wang W, Sylvester JT, Shimoda LA (2005) Chronic hypoxia inhibits Kv channel gene expression in rat distal pulmonary artery. Am J Physiol Lung Cell Mol Physiol 288:L1049-L1058

18. Wang J, Juhaszova M, Rubin LJ, Yuan XJ (1997) Hypoxia inhibits gene expression of voltage-gated $\mathrm{K}^{+}$channel alpha subunits in pulmonary artery smooth muscle cells. J Clin Invest 100:2347-2353
19. Conforti L, Bodi I, Nisbet JW, Millhorn DE (2000) $\mathrm{O}_{2}$-sensitive $\mathrm{K}^{+}$channels: role of the Kv1.2-subunit in mediating the hypoxic response. J Physiol 524 Pt 3:783-793

20. Archer SL, London B, Hampl V et al (2001) Impairment of hypoxic pulmonary vasoconstriction in mice lacking the voltagegated potassium channel Kv1.5. FASEB J 15:1801-1803

21. Patel AJ, Lazdunski M, Honore E (1997) Kv2.1/Kv9.3, a novel ATP-dependent delayed-rectifier $\mathrm{K}^{+}$channel in oxygen-sensitive pulmonary artery myocytes. EMBO J 16:6615-6625

22. Osipenko ON, Tate RJ, Gurney AM (2000) Potential role for Kv3.1b channels as oxygen sensors. Circ Res 86:534-540

23. Wang D, Youngson C, Wong V et al (1996) NADPH-oxidase and a hydrogen peroxide-sensitive $\mathrm{K}^{+}$channel may function as an oxygen sensor complex in airway chemoreceptors and small cell lung carcinoma cell lines. Proc Natl Acad Sci USA 93:13182-13187

24. Perez-Garcia MT, Lopez-Lopez JR, Gonzalez C (1999) Kvbeta1.2 subunit coexpression in HEK293 cells confers $\mathrm{O}_{2}$ sensitivity to Kv4.2 but not to Shaker channels. J Gen Physiol 113:897-907

25. Burhop KE, Selig WM, Malik AB (1988) Monohydroxyeicosatetraenoic acids (5-HETE and 15-HETE) induce pulmonary vasoconstriction and edema. Circ Res 62:687-698

26. Preston IR, Hill NS, Warburton RR, Fanburg BL (2006) Role of 12-lipoxygenase in hypoxia-induced rat pulmonary artery smooth muscle cell proliferation. Am J Physiol Lung Cell Mol Physiol 290:L367-L374 